\title{
An accurate model for simulating energetic behavior of PV grid connected inverters
}

\author{
Luis Davila-Gomez Antonio Colmenar-Santos Mohamed Tawfik Manuel Castro-Gil
}

\begin{abstract}
A B S T R A C T
This paper proposes a new model for characterizing the energetic behavior of grid connected PV inverters. The model has been obtained from a detailed study of main loss processes in small size PV inverters in the market. The main advantage of the used method is to obtain a model that comprises two antagonistic features, since both are simple, easy to compute and apply, and accurate. One of the main features of this model is how it handles the maximum power point tracking (MPPT) and the efficiency: in both parts the model uses the same approach and it is achieved by two resistive elements which simulate the losses inherent to each parameter. This makes this model easy to implement, compact and refined. The model presented here also includes other parameters, such as start threshold, standby consumption and islanding behavior. In order to validate the model, the values of all the parameters listed above have been obtained and adjusted using field measurements for several commercial inverters, and the behavior of the model applied to a particular inverter has been compared with real data under different working conditions, taken from a facility located in Madrid. The results show a good fit between the model values and the real data.

As an example, the model has been implemented in PSPICE electronic simulator, and this approach has been used to teach grid-connected PV systems. The use of this model for the maintenance of working PV facilities is also shown.
\end{abstract}

\section{Introduction}

Simulation of photovoltaic systems is becoming more and more important. There are a wide variety of software packages that allow you to make easier the processes of design and analysis of grid connected photovoltaic systems.

Referring to those focused on the design, we can find tools for the economic calculation of the systems, tools for dimensioning of the components, and those that integrate both economics and sizing issues.

The software programs for economic analysis allow you to calculate the total cost of photovoltaics systems, because they contain a database of commercial elements and their market prices. If the software includes the calculation of the energy generated, it will allow us to determine the payback time of the installation and thus calculate investment feasibility. These programs perform relatively simple calculations, and require little interaction with the user. In this group we can find RETScreen [1] and PVSYST [2]. 
For the sizing of photovoltaic systems, some companies have developed their own programs, mostly in spreadsheets, which allow them to undertake their projects of PV systems, but there are other programs that perform this function [3].

Referring to those focused on the analysis, we found that some of them simulate the generation of the system, others estimate shading losses, others help to optimize the system, etc.

PV simulation programs take data from a system already sized and provide a detailed time analysis of its operation. This analysis allows us to verify the sizing done, to check the impact on production of a malfunction or a voluntary stop of the system, to test the performance under different conditions, etc. Among others, the most used are: HOMER [4], which allows the simulation of hybrid systems, PV-DesignPro [5], and PVSYST.

On the other hand, generic simulators are those designed for the study of different types of problems. Most of them also allow simulations of photovoltaic systems, both stand-alone and grid-connected. There are a wide variety of tools of this type, and there have been published papers where you can find examples of applications in photovoltaic systems; for example with the programming languages $\mathbf{R}$ [6] or JAVA [7], or in commercial programs for electrical simulation [8-10]. From those used for photovoltaic simulation we can highlight two:

- SIMULINK is suitable when you want to simulate the behavior of systems with photovoltaic and mechanical parts such as an electric vehicle, as in [11]. But it may also be applied to the simulation of control strategies in photovoltaic systems [12], to the performance evaluation of the MPPT of the inverter [13,14], to the improvement in the design of PV inverters [16] and to simulate the behavior of PV cells and modules [17,18]. SIMULINK is also useful for full modeling of stand-alone, grid-connected and hybrid photovoltaic systems [19], and allows identifying the system using parameters extraction methods from real measurements and diagnosing potential faults [20], which helps in the maintenance of the systems. We also found examples of application in the area of the photovoltaic systems teaching in [21].

- SPICE simulation environment is the mostly used in electronics. Also its use for the simulation of photovoltaic systems has a long history. There are a vast literature on power converters simulations [22,23], the study of their faults [24] and the MPPT [25] to validate its use in photovoltaic systems. In regard to the photovoltaic elements, we can find models of the solar cell [26] and the solar panel [27], these models can help to study the effect of shading and propose solutions [28], to determine the need for the use of bypass and blocking diodes [29,30]. In academia and for teaching purposes the simulator was used to study the performance of solar cells and panels [26] and there are tools to simulate photovoltaic systems in Undergraduate and Master Degree courses [31].

There are two different kinds of models to simulate a photovoltaic inverter:

1. Topological models, which includes the whole electric scheme of the inverter, or a simplified version of it, as in [8$10,12,15,16,23,24,31]$. These models allow detailed short time simulations and they are very useful for the electronics design. However, usually the models are too complex and only can simulate the inverters for which they were created, and have a little use in PV simulation. They are more concerned with the long-term behavior of the system. These models are not related to the model proposed in this paper, thus it will not be studied.

2. Behavioral models, which are focused on the input-output relationships and on the working principles of the inverter. These models are more generics and can simulate any inverter with the same operating principle. There are application examples in [22] for islanding behavior, for the maximum power point tracking (MPPT) in [13,14,25,31], and for the inverter efficiency in $[25,31,32]$.

The proposed model belongs to the group "behavioral models"; for this reason some of them are detailed next.

The MPPT model in [13] simulates the control strategy, which is different for every case of study. To simulate the efficiency, it uses only six values of the efficiency curve. A model is proposed in [14] with two main blocks: a DC/DC converter and a control circuit. In [25], a digital controller is developed for a MPPT based on "Perturbation \& Observation" method. Finally, [31] shows a model based on an ideal DC/DC converter with constant MPPT efficiency.

The efficiency model proposed in [25] is a lookup table with values depending on the input power. For intermediate values, a linear interpolation is used. The model presented in [31] considers a constant efficiency for the inverter, so the output power is oversized for low values of the input power. The one proposed in [32] is a losses model, similar to the proposed in this paper. Both will be compared in Section 3.2.

This paper focuses on the inverter of photovoltaic systems and their characterization. The rest of the paper is structured as follows. Section 2 describes a model for photovoltaic inverter that can be simulated with any of the simulators described above. Section 3 presents the methodology for the calculation and validation of the model. Section 4 shows a selection of examples based on the last application mentioned in the previous paragraph.

Finally, the drawn conclusions from this contribution are provided.

\section{Inverter model}

The inverter model developed can simulate several characteristics related with its behavior. These can be divided into two groups: Energetic performance and islanding protections. 


\subsection{Energetic performance characteristics}

In the energetic performance group, the mains elements are:

1. Efficiency, the ratio between AC output power and DC input power.

$$
\eta=\frac{P_{A C}}{P_{D C}}
$$

2. Start threshold; it is the minimum DC power needed to start the power conversion.

3. Standby consumption is the power wasted by the inverter when no power conversion is making.

4. Maximum power point tracking. The inverter circuits try to obtain all power of PV arrays, and this feature measures how near it is. It can be modeled as a new efficiency, and can be defined as the ratio between power obtained from the PV array by the inverter and theoretical maximum power available from the PV array.

$$
\eta_{M P P T}=\frac{\int P_{R E A L} d t}{\int P_{M A X} d t}
$$

\subsection{Energetic performance models}

A model has been developed that simulates the inverter efficiency based on inverter losses. Unlike behavioral models [25,31,32], this model is based on the one developed by Chivelet et al. [33,34] for stand-alone inverters. The model has been adapted here for grid connected inverters.

We can expect two kinds of losses in an inverter::

1. Losses due to the consumption of inverter circuits, those always are present when the power conversion is on. These losses are independent on input power.

2. Losses that depend on the amount of power conversion (commutation losses, wiring losses). These losses are strongly dependent on input power.

The action of both types of losses shapes the efficiency curve.

The model is made with an ideal inverter (the one with efficiency equal to one for all possible power output values), which has a serial input resistor and a parallel output resistor.

According to previous model description, the electrical scheme of this approach would be as shown in Fig. 1.

The efficiency can be calculated as follows:

$$
\eta=\frac{P_{A C}}{P_{D C}}=\frac{V_{A C} I_{A C}}{V_{D C} I_{D C}}
$$

To write the efficiency as function of input voltage and power output, you must get the value of $I_{D C}$. In order to get $I_{D C}$, you must take into consideration that in an ideal inverter power input equals to power output:

$$
V_{E} I_{D C}=V_{0} I_{0}=V_{A C} I_{0}
$$

This equation can be written as follows:

$$
V_{D C} I_{D C}-I_{D C}^{2} R_{S}=\frac{V_{A C}^{2}}{R_{P}}+V_{A C} I_{A C}
$$

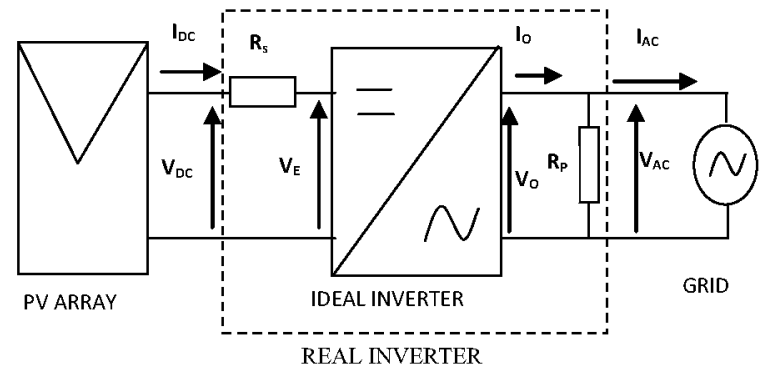

Fig. 1. Equivalent electric circuit of a grid connected inverter. 


$$
R_{S} I_{D C}^{2}-V_{D C} I_{D C}+\left(\frac{V_{A C}^{2}}{R_{P}}+P_{A C}\right)=0
$$

Solving for DC current:

$$
I_{D C}=\frac{V_{D C} \pm \sqrt{V_{D C}^{2}-4 R_{S}\left(\frac{V_{A C}^{2}}{R_{P}}+P_{A C}\right)}}{2 R_{S}}
$$

From the two possible solutions, we took the one with negative sign before the square root, and calculating we obtain:

$$
I_{D C}=\frac{V_{D C}-V_{D C} \sqrt{1-4 \frac{R_{S}}{V_{D C}^{2}}\left(\frac{V_{A C}^{2}}{R_{P}}+P_{A C}\right)}}{2 R_{S}}
$$

That introduced in Eq. (3) gives us:

$$
\eta=\frac{P_{A C}}{V_{D C} I_{D C}}=\frac{P_{A C}}{V_{D C}\left[\frac{V_{D C}-V_{D C} \sqrt{1-4_{V_{D C}^{2}}^{R_{S}}\left(\frac{V_{A C}^{2}}{R_{P}}+P_{A C}\right)}}{2 R_{S}}\right]}
$$

Thus, the final equation for the efficiency is as follows:

$$
\eta=\frac{2 R_{S} P_{A C}}{V_{D C}^{2}\left(1-\sqrt{1-4 \frac{R_{S}}{V_{D C}^{2}}\left(\frac{V_{A C}^{2}}{R_{P}}+P_{A C}\right)}\right)}
$$

Eq. (10) gives us the efficiency as a function of DC input voltage, RMS AC output voltage and output power, and includes the two resistive parameters: $R_{S}$ and $R_{P}$. These resistive parameters represent the inverter losses.

$R_{S}$ stands for coupling losses between $D C$ and $A C$ side of the inverter. Fig. 2 shows the variation of the efficiency when the value of this parameter is modified. In the figure, the more value of $R_{S}$, the less value of efficiency in the flat zone of the graphic, and as you can see, the shape of the graphic is not affected by the variation of this parameter. $R_{S}$ is a serial resistor placed on the input so that power losses due to this component increases when input power increases. Thus, the higher the output power, the higher the contribution of $R_{S}$ to efficiency. Thereby, $R_{S}$ is related to the losses that depend on the input power, such as commutation losses.

The $R_{p}$ parameter is responsible of the low power zone shape, as we can see in Fig. 3. As you can see, the higher the value of $R_{p}$, the higher the slope of the curve, and if $R_{p}$ is high enough the efficiency becomes almost ideal $(\eta=1$ for all possible power output values). $R_{p}$ is a parallel resistor in the output stage of the inverter; power losses on this component are proportional to the square of RMS AC output voltage. Output voltage is equal to grid voltage, and then loss on $R_{p}$ will be a constant quantity over all the output power range. Then, $R_{P}$ represents operation losses of inverter. This means that the influence of $R_{p}$ will be very important in low power zone of efficiency, decreasing as power output increases.

The inverter efficiency does not have valid values below the start threshold. The curves obtained with the efficiency model have values not equal to zero when output power is near to zero because the model does not include the start threshold characteristic of the inverter. However, the start threshold can be easily included if you modify Eq. (10) in this way:

$$
\begin{aligned}
& \eta=0 \text { if } P_{D C}<P_{A} \\
& \eta=\frac{2 R_{S} P_{A C}}{V_{D C}^{2}\left(1-\sqrt{1-4 \frac{R_{S}}{V_{D C}^{2}}\left(\frac{V_{A C}^{2}}{R_{P}}+P_{A C}\right)}\right)} \text { if } P_{D C}>P_{A}
\end{aligned}
$$

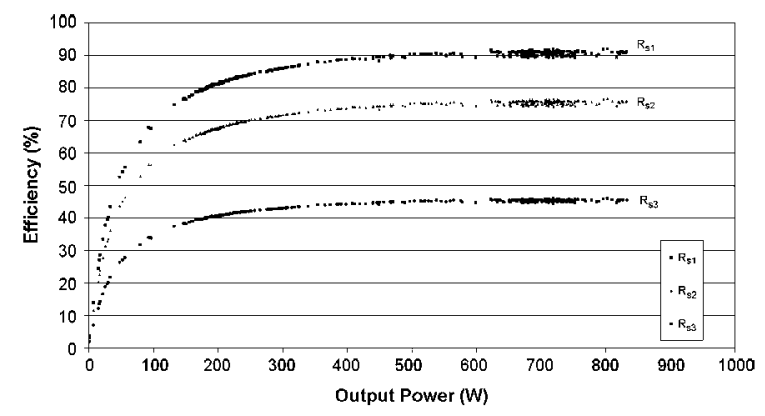

Fig. 2. Efficiency variation as a function of $R_{S}$ parameter $\left(R_{S 1}<R_{S 2}<R_{S 3}\right)$. 


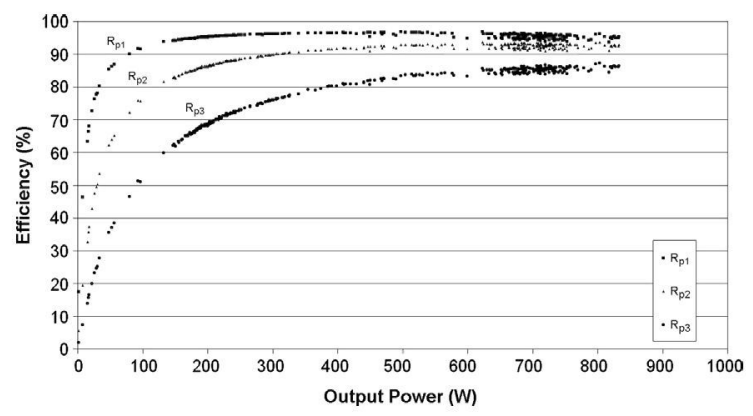

Fig. 3. Efficiency variation as a function of $R_{P}$ parameter $\left(R_{P 1}>R_{P 2}>R_{P 3}\right)$.

where $P_{A}$ is the input power necessary to the inverter to start the conversion.

The efficiency model proposed in this work is different from those pointed in Section 1. In $[13,25]$ the models are limited to a few specific values, while the proposed can cover the whole curve. In [31] the efficiency is a constant; in contrast, in the proposed model the efficiency varies with inverter parameters. Finally, the model from Jantsch et al. [32] is the most similar to the proposed, but it needs three parameters instead of the two resistive parameters presented here. This model will be compared with the proposed in Section 3.

When the inverter stops energizing the utility (for example, when the DC power remains below the start threshold), there are an amount of power consumption from the grid, that is called the standby consumption. To model this characteristic, you can use a resistor $R_{S C}$ to simulate this power like a loss. Then, the value of $R_{S C}$ will be as follows:

$$
R_{S C}=\frac{V_{A C}^{2}}{P_{S C}}
$$

where $P_{S C}$ is the power needed in the standby mode of the inverter.

This resistor will replace the parallel output resistor $R_{P}$ when the inverter enters in standby mode, while efficiency is equal to zero. This part of the model is shown in Fig. 4.

The last characteristic for the model is the maximum power tracking. The parameter we will use to determine the performance of a maximum power point tracker is the efficiency [14], as in Eq. (2). But if we define instantaneous power efficiency as in [35]:

$$
\eta_{\text {MPPT }}=\frac{P_{D C}}{P_{\text {MAX }}}
$$

This efficiency depends on the input power, and you can find two different losses in this parameter:

- On the one hand, low power losses, which are relatively high due to the difficulty in determining the optimum operating point when the power curve is very flat.

- Moreover, for high power values the tracker has very small losses, since the perfect track does not exist.

The combination of both types of losses makes the instantaneous power efficiency curve. This one is very similar to the efficiency curve of the inverter, and is simulated using a similar model.

We will simulate MPPT losses using two resistors, one in series with the input and another in parallel with the output of an ideal MPPT, without any loss. This is a new model with the physical sense of a loss model, as opposed to purely mathematical models [36,37] or those based on the topology of the electronic circuitry [13] or its behavior [38]. According to the description of the model, the scheme would be the one shown in Fig. 5.

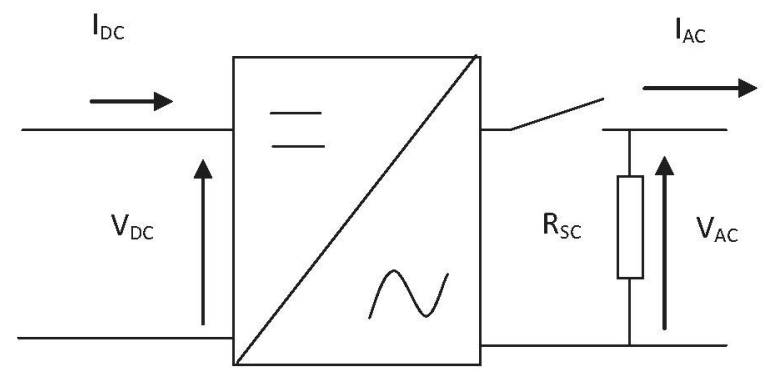

Fig. 4. Standby consumption part of the model. 
The MPPT efficiency can be calculated in the same way as inverter efficiency. Then, we can write Eq. (14) as follows:

$$
\eta_{M P P T}=\frac{P_{D C}}{P_{M A X}}=\frac{V_{D C} I_{D C}}{V_{M A X} I_{M A X}}
$$

For the ideal MPPT:

$$
V_{I} I_{M A X}=V_{0} I_{0}=V_{D C} I_{0}
$$

To obtain $I_{\text {MAX: }}$

$$
\begin{aligned}
& V_{M A X} I_{M A X}-I_{M A X}^{2} R_{S M}=\frac{V_{D C}^{2}}{R_{P M}}+V_{D C} I_{D C} \\
& R_{S M} I_{M A X}^{2}-V_{M A X} I_{M A X}+\left(\frac{V_{D C}^{2}}{R_{P M}}+P_{D C}\right)=0 \\
& I_{M A X}=\frac{V_{M A X} \pm \sqrt{V_{M A X}^{2}-4 R_{S M}\left(\frac{V_{D C}^{2}}{R_{P M}}+P_{D C}\right)}}{2 R_{S M}}
\end{aligned}
$$

From the two possible solutions, we took the one with negative sign before the square root, and calculating we obtain:

$$
I_{M A X}=\frac{V_{M A X}-V_{M A X} \sqrt{1-4 \frac{R_{S M}}{V_{M A X}^{2}}\left(\frac{V_{D C}^{2}}{R_{P M}}+P_{D C}\right)}}{2 R_{S M}}
$$

Thus, from Eq. (15), the final formula for the MPPT efficiency can be written as follows:

$$
\eta_{M P P T}=\frac{2 R_{S M} P_{D C}}{V_{M A X}^{2}\left(1-\sqrt{1-4 \frac{R_{S M}}{V_{M A X}^{2}}\left(\frac{V_{D C}^{2}}{R_{P M}}+P_{D C}\right)}\right)}
$$

Eq. (15) includes two resistive parameters: $R_{S M}$ and $R_{P M}$. $R_{S M}$ represents the high power losses. $R_{S M}$ is a resistor connected in series with the input so that the power loss on it increases with the value of the input power. Its contribution to the shape of the MPPT efficiency is the same as shown in Fig. 2 for $R_{S}$.

$R_{P M}$ is the parameter for low power losses. If $R_{P M}$ value increases, the slope of the curve increases too, and for high values of the resistive parameter, the MPPT efficiency is near the ideal value ( $\eta_{M P P T}=1$ for any output power). The resistor is in parallel with the output; then the losses depend on the square of the output voltage. Since the output voltage varies mainly with the variation of temperature, the loss due to this resistor varies very slowly. Its influence on MPPT efficiency is very important in the low power zone, and the shape of the curve is modified in the same way as $R_{P}$ in Fig. 3.

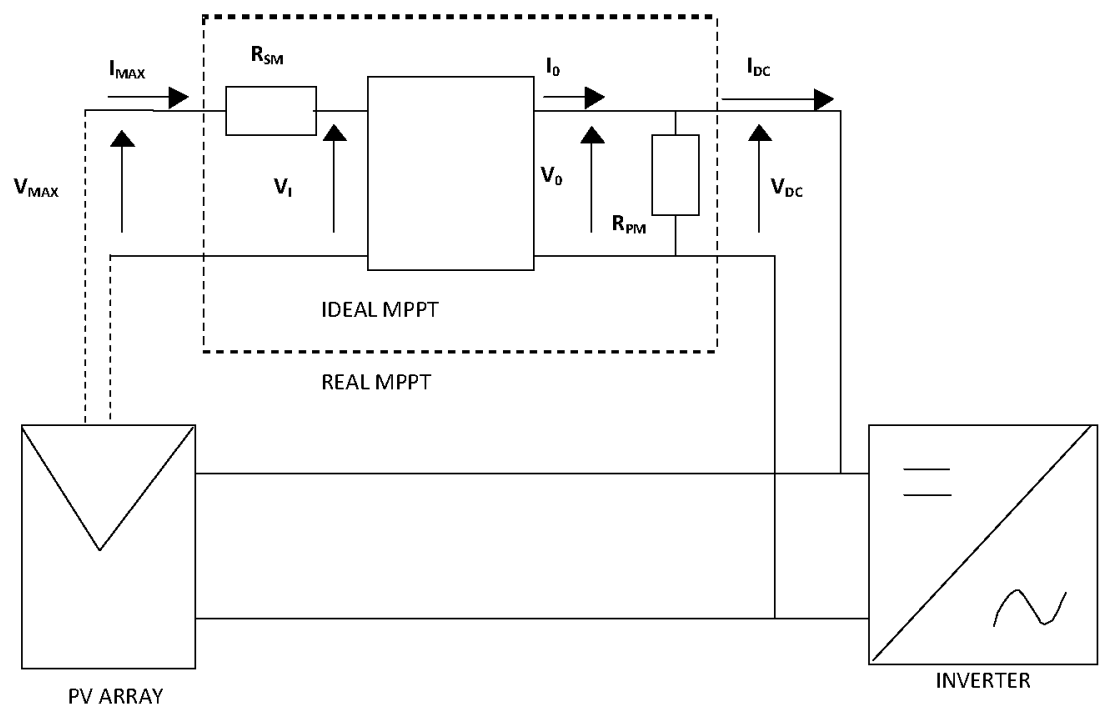

Fig. 5. Equivalent electric circuit of the MPPT of a grid connected inverter. 
The MPPT efficiency model proposed in this work is different from those pointed in Section 1. Most models are based on simulating one control strategy $[13,14,25]$ and then are only suitable for inverters using these control strategies. In [31], MPPT is a constant; in contrast to this new model, which has a MPPT efficiency that varies with inverter parameters.

\subsection{Quality characteristics}

This set of characteristics evaluates if the signal is within the operating range. For the proposed model, we use the following:

1. Over voltage: it is the value of the utility voltage above a certain threshold.

2. Under voltage: by analogy, the under voltage is a voltage value on the utility below a set limit.

If there is one of these situations, the inverter should stop energizing the grid.

\subsection{Quality models}

The model used to simulate these characteristics is very simple. We only make a comparison between RMS present value of grid voltage and the limits set according to standard IEC 61727. When the comparison shows an out of limit situation, we force the inverter to the standby mode.

Thus, the model will have two parameters: the lower limit of voltage, $V_{\min }$ and the upper one, $V_{\max }$.

\subsection{Electrical security characteristics}

In our case, the most relevant feature is the islanding behavior. Islanding is a state in which a portion of the electric utility grid, containing load and generation, continues to operate isolated from the rest of the grid. This represents a potential safety hazard of a line worker coming in contact with an energized line that is presumed to be de-energized [39].

Any grid-connected inverter has to detect island conditions and should cease to energize the public electric power grid under specific conditions. This conditions and the test procedure are the purpose of the IEC 62116 standard. The inverters that meet the requirements of IEC 62116 and IEC 61727 can be considered non-islanding.

\subsection{Electrical security model}

In our model, to simulate the islanding behavior, we force the inverter to enter the standby mode when a loss of power occurs. This situation is detected with the quality model. The inverter shall not reconnect before the frequency and voltage have been maintained within the specified limits for at least the time addressed in IEC 61727.

So the model needs only one parameter: the reconnection time $T_{R}$.

The electrical security model and the quality model are interconnected, because both have to force the inverter to enter the standby mode. Therefore, both models generate a condition of "standby mode" that must be combined to generate the correct alarm.

Table 1 shows all the parameters required for the different parts of this model.

\section{Model calculation}

To implement the inverter model developed, it is necessary to obtain the numerical values of the parameters for a particular device. Some of them can be found in its data sheet, or can be calculated from this information. Using the parameters calculated for several inverters we can validate our model.

Table 1

Parameters for the model.

\begin{tabular}{ll}
\hline Characteristic & Parameter(s) \\
\hline Energetic performance & \\
Efficiency & $R_{S}, R_{P}$ \\
Start threshold & $P_{A}$ \\
Standby consumption & $R_{S C}$ \\
MPPT & $R_{S M}, R_{P M}$ \\
Ouality & \\
Over and under voltage & $V_{\min }, V_{\max }$ \\
Electrical security & \\
lslanding behavior & $T_{R}$ \\
\hline
\end{tabular}




\subsection{Parameters extraction}

In Table 1 we have all the parameters for the model. The nominal values of many of them can be found in the data sheet of the inverter. We can find values for $P_{A}, V_{\min }, V_{\max }$ and $T_{R}$. On the other hand, $R_{S C}$ can be calculated from $P_{S C}$, included in the data sheet. But dissipative parameters are not included in data sheets, and must be calculated.

There are four dissipative parameters: $R_{S}, R_{P}$ for the overall efficiency and $R_{S M}, R_{P M}$ for the MPPT efficiency. Methods for calculating $R_{S}$ and $R_{P}$ are the same as for $R_{S M}$ and $R_{P M}$ because we use a similar approach.

To calculate $R_{S}$, we take Eq. (5) and solve for this parameter:

$$
R_{S}=\frac{V_{D C} I_{D C}-\frac{V_{A C}^{2}}{R_{P}}-V_{A C} I_{A C}}{I_{D C}^{2}}
$$

When input and output powers are high, the $R_{P}$ losses term can be ignored. Then:

$$
R_{S} \approx \frac{P_{D C}-P_{A C}}{I_{D C}^{2}}
$$

In order to evaluate $R_{S}$ we must use a set of values for $P_{D C}, P_{A C}$ and $I_{D C}$ as higher as possible, between ones obtained in measures.

To evaluate $R_{P}$, we take again Eq. (5) and find now $R_{P}$ :

$$
\frac{1}{R_{P}}=\frac{V_{D C} I_{D C}-I_{D C}^{2} R_{S}-V_{A C} I_{A C}}{V_{A C}^{2}}
$$

When input and output powers are low, the $R_{S}$ losses term can be ignored this time. Then, we obtain:

$$
\frac{1}{R_{P}} \approx \frac{P_{D C}-P_{A C}}{V_{A C}^{2}}
$$

Several test showed that the most suitable set of values $P_{D C}, P_{A C}$ and $V_{D C}$ for calculating $R_{P}$ are those nearest to the zone of slope change in efficiency curve [40].

Likewise, to calculate $R_{S M}$, we take now Eq. (17) and solving:

$$
R_{S M}=\frac{V_{M A X} I_{M A X}-\frac{V_{D C}^{2}}{R_{P M}}-V_{D C} I_{D C}}{I_{M A X}^{2}}
$$

And for high values of input and output power, the solution can be approximated to:

$$
R_{S M} \approx \frac{P_{M A X}-P_{D C}}{I_{M A X}^{2}}
$$

To evaluate $R_{P M}$, we take again Eq. (17) and we arrange it as follows:

$$
\frac{1}{R_{P M}}=\frac{V_{M A X} I_{M A X}-I_{M A X}^{2} R_{S M}-V_{D C} I_{D C}}{V_{D C}^{2}}
$$

In the low power zone, the $R_{S M}$ losses term can be ignored, and we obtain:

$$
\frac{1}{R_{P M}} \approx \frac{P_{M A X}-P_{D C}}{V_{D C}^{2}}
$$

As in the efficiency sub model, the most suitable set of values $P_{M A X}, P_{D C}$ and $V_{D C}$ for calculating $R_{P M}$ are those nearest to the zone of slope change in MPPT efficiency curve.

To compute the parameters values, measured efficiency data from inverters were used. As shown in Fig. 6, for every value of output power we have several values of efficiency, and therefore various parameters values. To obtain a single value for each parameter, an algorithm was created that uses the values of $R_{S}$ and $R_{P}$ (or $R_{S M}$ and $R_{P M}$ ) obtained to calculate the efficiency curve for different combinations of these parameters values. These curves are compared with the actual curve and the algorithm chooses the one with a better correlation coefficient.

\subsection{Model validation}

To validate the efficiency part of the model, we need to measure $I_{D C}, V_{D C}, P_{D C}, V_{A C}$ and $P_{A C}$, and extract the parameters $R_{S}$ and $R_{P}$ using the method described in Section 3.1. The block diagram of the experimental test setup used to measure is shown in Fig. 7. The inverter input current and voltage are measured using channel A of a precision power analyzer, and channel B is used for output values. The records were made in sunny days, taking at least two days of measuring. The computer stores and processes the data. 


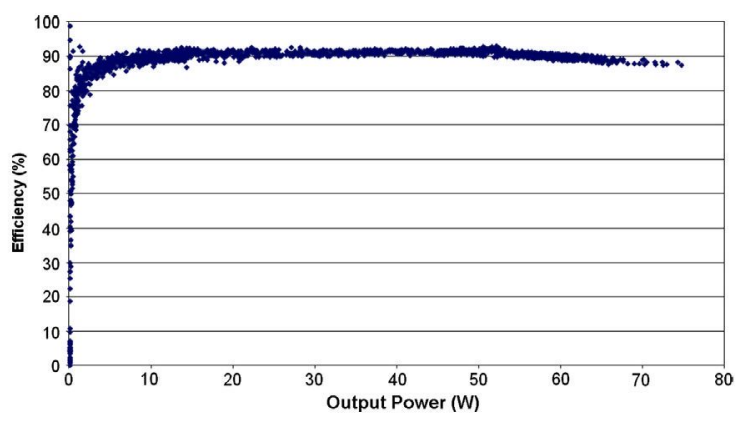

Fig. 6. Measured curve of efficiency for a commercial inverter.

With $P_{D C}$ and $P_{A C}$, we can calculate $\eta$ using (3). Then, with $I_{D C}$ and $V_{A C}$ we find the seeds for $R_{S}$ and $R_{P}$ with (27) and (29). The extraction algorithm uses all these measured values and also $V_{D C}$. With the final values for $R_{S}$ and $R_{P}$, we can calculate again $\eta$ using now (10), and compare both values with two statistics index:

1. Pearson's correlation, that gives the quality of the curve fitting between the calculated efficiency and the measured data.

2. Root mean square error (RMSE), as a measure of standard deviation.

These statistics have been widely used in similar works $[20,41]$.

The model has been verified with a set of inverters at the Industrial Technical Engineering School of the Polytechnic University of Madrid (ETSIDI - UPM) and in the Laboratory of Photovoltaic Systems at the Research Centre for Energy, Environment and Technology (CIEMAT).

For the inverters at ETSIDI-UPM, the results are shown in Figs. 8 and 9 and Table 2.

For comparison, Figs. 8 and 9 shows Jantsch et al. model [32] in addition to the proposed model. In this model, efficiency depends on the self-consumption, the load dependent losses, and the output power by the equation:

$$
\eta=\frac{P_{\text {out }}}{P_{\text {out }}+k_{0}+k_{1} P_{\text {out }}+k_{2} P_{\text {out }}^{2}}
$$

where the self-consumption are represented by $k_{0}$, the load linear proportional losses by $k_{1}$ and the load square proportional losses by $k_{2}$.

As can be seen, there are a good fit between the real data and the models. However, Jantsch model for inverter A shows a RMSE of $2.32 \%$, almost twice that the proposed model. For inverter B, Eq. (30) shows a better fit than the new model (Eq. (10)), but for this good fit it has been necessary to adjust the $k_{0}$ parameter from the measured one $(0.16 \%)$ to an out of range $1.33 \%$, so that the values cannot be considered valid. Using the correct value for $k_{0}$, the RMSE reaches $5.6 \%$. As demonstrated, the fitting between the proposed model and measured efficiency is more accurate than with other models.

For the inverters at CIEMAT, Table 3 is a summary of the parameters and statistics, and the graphical results are shown in Figs. 10-12.

To validate the efficiency in tracking the maximum power point, you need to measure $V_{D C}, P_{D C}, G$ and $T$ (the latter parameters are necessary to calculate $I_{M A X}, V_{M A X}$ and $P_{M A X}$ using equation shows in [31]) and extract the parameters $R_{S M}$ and $R_{P M}$ as described in Section 3.1. The measuring system can be the same to the one shown in Fig. 7. The procedure is virtually identical to that used to validate the efficiency.

Testing was performed using one of the inverters installed at ETSIDI/UPM. The parameters and errors indicated in Table 4 were obtained, and the graph of Fig. 13 summarizes the results.

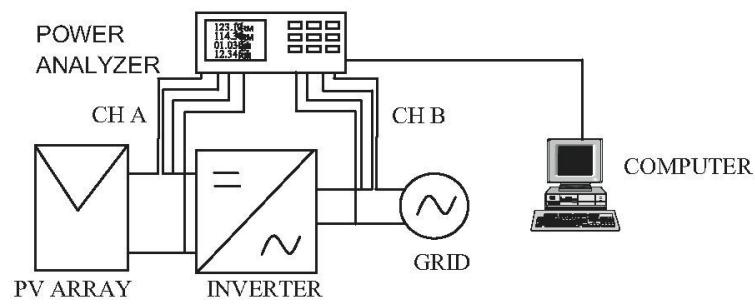

Fig. 7. Block diagram of the experimental test setup for the evaluation of the conversion efficiency. 


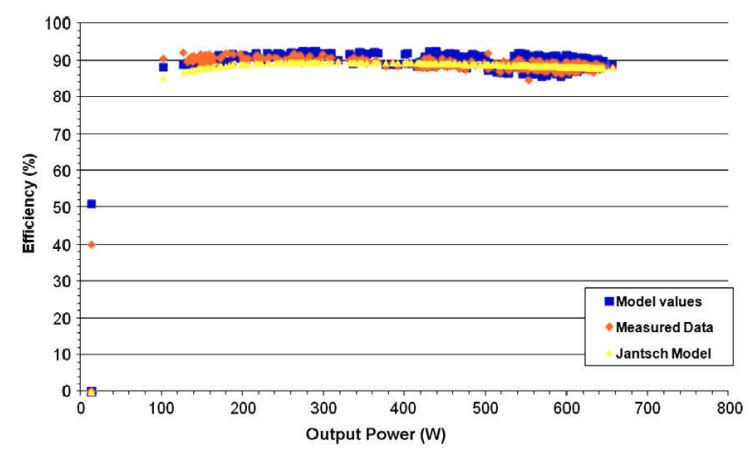

Fig. 8. Efficiency of the inverter "A" at EUITI-UPM in terms of output power. Orange diamonds are experimental data, blue squares are model values and yellow triangles are Jantsch model values. (For interpretation to colors in this figure, the reader is referred to the web version of this paper.)

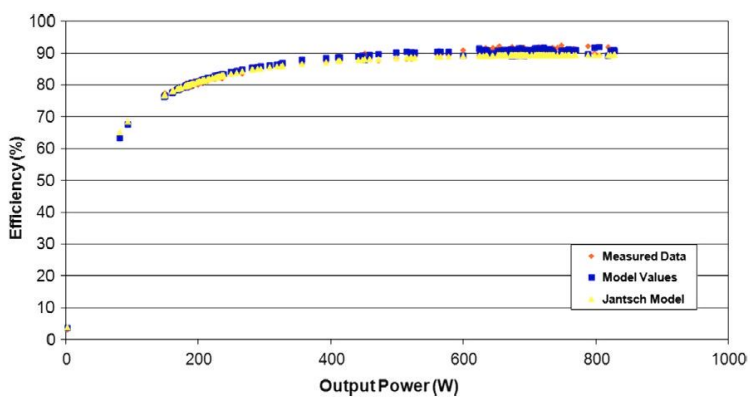

Fig. 9. Efficiency of the inverter " $\mathrm{B}$ " at EUITI-UPM in terms of output power. Orange diamonds are experimental data, blue squares are model values and yellow triangles are Jantsch model values. (For interpretation to colors in this figure, the reader is referred to the web version of this paper.)

Table 2

Numerical results for inverters at EUITI-UPM.

\begin{tabular}{llllll}
\hline Inverter & Nominal power $(\mathrm{W})$ & $R_{S}(\boldsymbol{\Omega})$ & $R_{P}(\boldsymbol{\Omega})$ & Pearson's correlation & RMSE $(\%)$ \\
\hline A & 850 & 0.26 & 4353 & 0.99888 & 1.25 \\
B & 3000 & 0.6 & 1200 & 0.99958 & 0.98 \\
\hline
\end{tabular}

Table 3

Numerical results for inverters at CIEMAT.

\begin{tabular}{llllll}
\hline Inverter & Nominal power $(\mathrm{W})$ & $R_{S}(\Omega)$ & $R_{P}(\Omega)$ & Pearson's correlation & RMSE $(\%)$ \\
\hline $\mathrm{C}$ & 700 & 2.6 & 5498 & 0.99426 & 1.57 \\
$\mathrm{D}$ & 850 & 1.2 & 3004 & 0.99986 & 0.77 \\
$\mathrm{E}$ & 2000 & 2.8 & 2224 & 0.99702 & 1.39 \\
\hline
\end{tabular}

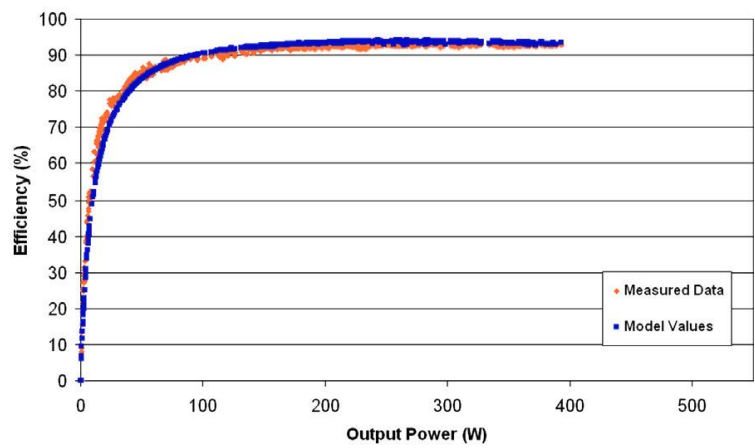

Fig. 10. Efficiency of the inverter "C" at CIEMAT in terms of output power. 


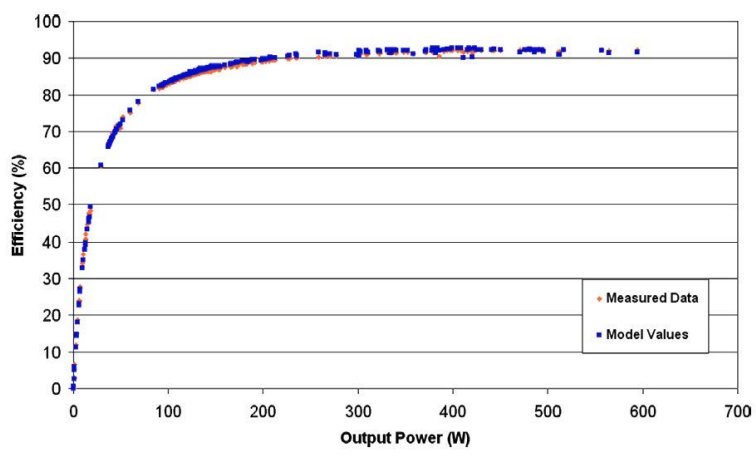

Fig. 11. Efficiency of the inverter "D" at CIEMAT in terms of output power.

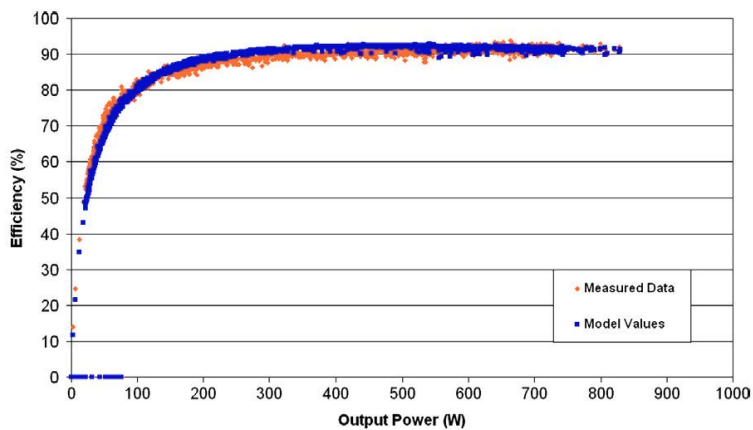

Fig. 12. Efficiency of the inverter "E" at CIEMAT in terms of output power.

In addition, measurements were made with the 3 inverters installed at CIEMAT. Figs. 14-16 show the graphs of the modeled and real MPPT efficiency of such inverters, showing the small dispersion between the values obtained by modeling the inverters and the curves obtained with actual measurements.

The values of the dissipative parameters are shown in Table 5.

In this table we can see that for these inverters, the $R_{S M}$ resistance ranges between 0.18 and $5.4 \Omega$ and $R_{P M}$ varies between 423 and $1802 \Omega$.

Table 4

Parameters values and statistical error from 'B' inverter.

\begin{tabular}{llllll}
\hline Inverter & Nominal power $(\mathrm{W})$ & $R_{S M}(\Omega)$ & $R_{P}(\Omega)$ & Pearson's correlation & RMSE $(\%)$ \\
\hline B & 3000 & 1.1 & 267 & 0.9966 & 2.7 \\
\hline
\end{tabular}

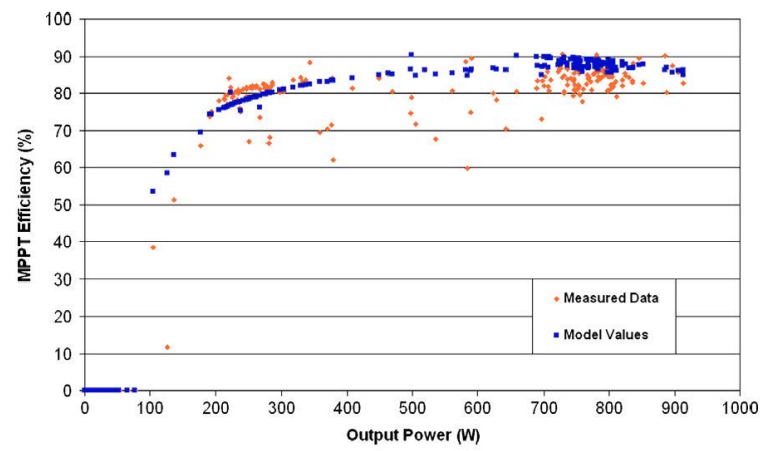

Fig. 13. MPPT efficiency for ' $\mathrm{B}$ ' inverter. Orange diamonds are experimental data, and blue squares are model values (For interpretation of the references to colors in this figure legend, the reader is referred to the web version of this paper) 


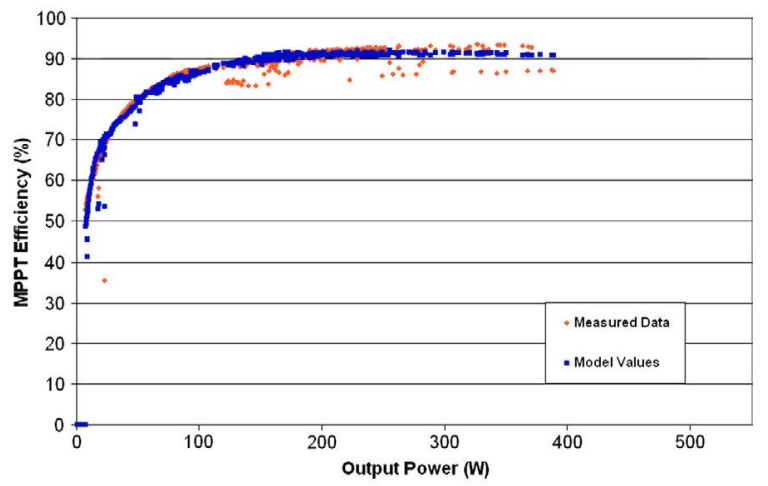

Fig. 14. MPPT efficiency for ' $\mathrm{C}$ ' inverter.

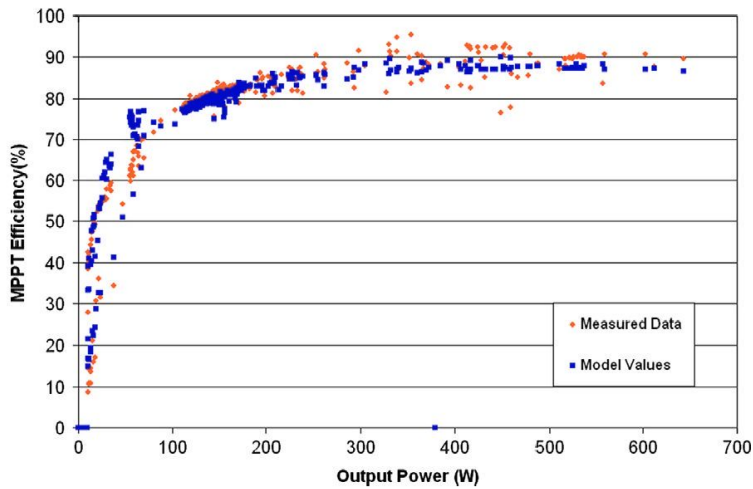

Fig. 15. MPPT efficiency for ' $D$ ' inverter.

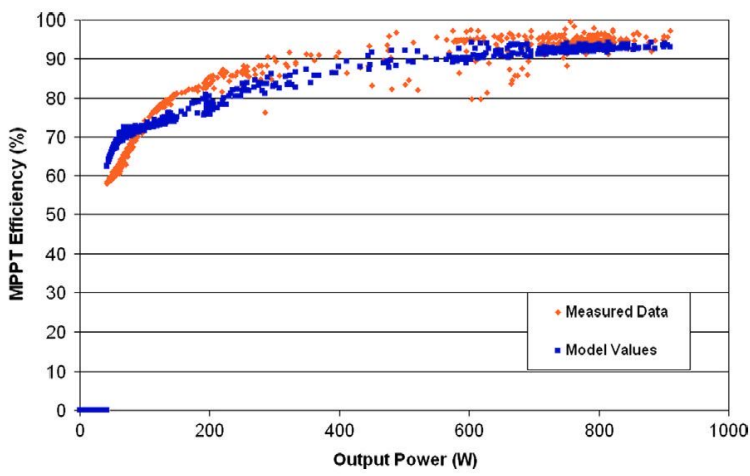

Fig. 16. MPPT efficiency for ' $E$ ' inverter.

Table 5

$R_{S M}$ and $R_{P M}$ parameters for inverters at CIEMAT.

\begin{tabular}{llllll}
\hline Inverter & Nominal power $(W)$ & $R_{S M}(\Omega)$ & $R_{P M}(\Omega)$ & Pearson's correlation & RMSE $(\%)$ \\
\hline C & 700 & 5.4 & 1802 & 0.9942 & 1.98 \\
D & 850 & 2.3 & 423 & 0.9981 & 1.69 \\
E & 2000 & 0.18 & 673 & 0.9983 & 2.20 \\
\hline
\end{tabular}

In terms of error, the modeling of the MPPT efficiency exhibits errors greater than those obtained in the modeling of the efficiency, but largely coincides with what has been achieved experimentally, the error reaching the value of $3.4 \%$ in the worst case. Compared with the model in [31], which uses a constant value for MPPT efficiency, the error is even greater, in the range of $15 \%$. 
With this in mind, we can conclude that, although not as closely as for efficiency, the proposed model for efficiency on maximum power point tracking of a PV inverter correctly simulates this characteristic, and it can be implemented in a straightforward manner to be a part of this model for energy performance of inverters.
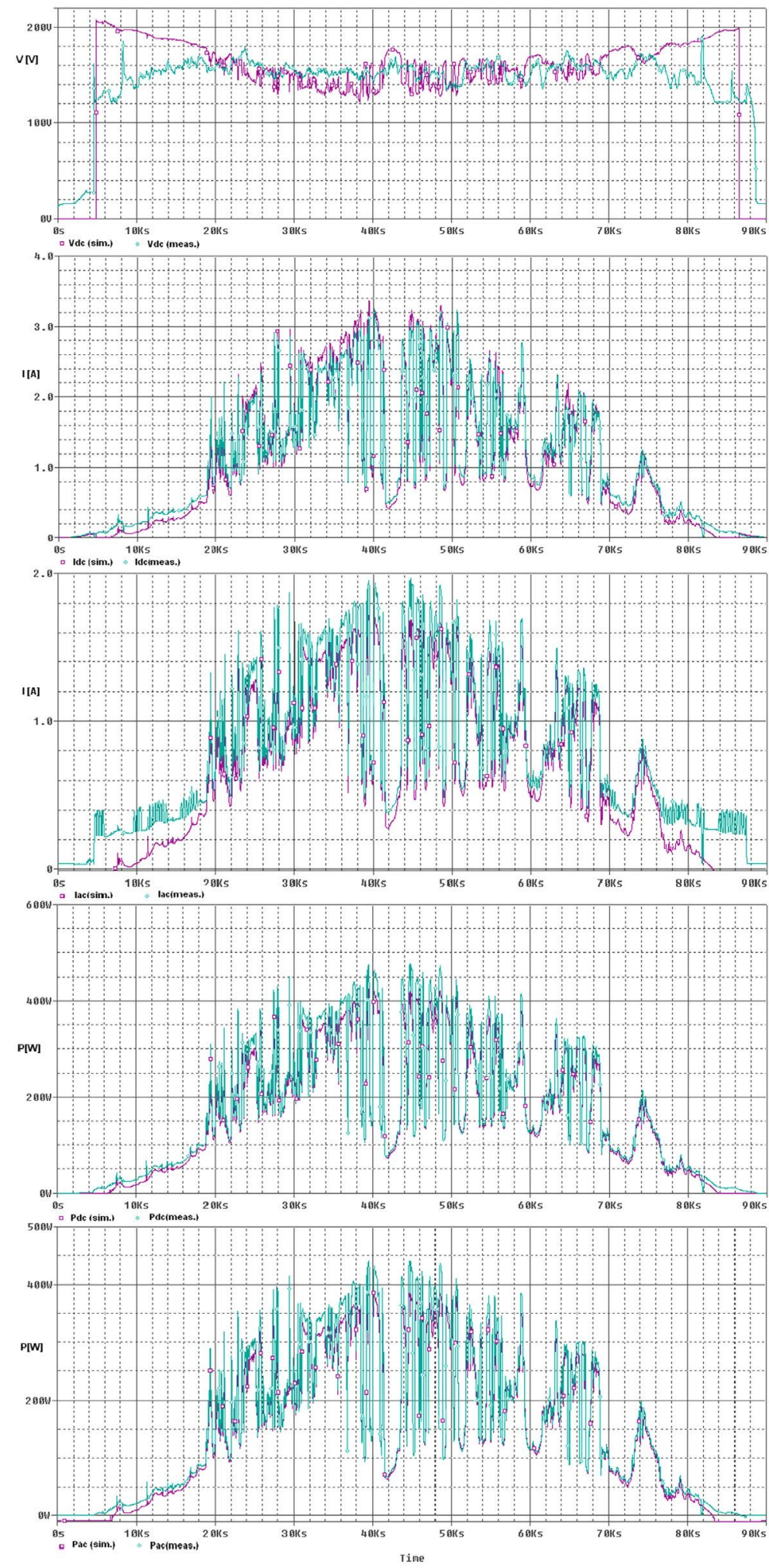

Fig. 17. Input and output voltages, currents and powers on an inverter over a day. 


\section{Results and applications}

The accuracy of the model presented here can be verified by comparing the values of a simulation with real data. Fig. 17 shows the comparison between simulated and actual values for input and output voltages and currents, and for input and output power of an inverter. The graph shows in purple color the curves corresponding to the values of the simulation, and in green color, the curves with the measured data. The upper part of the graph shows the DC voltage; we can see that the greatest differences are for low power values. In the middle we have the curves corresponding to the DC and AC currents, with a good fit between them. The lower part shows the input and output power of the inverter; being a losses model, the best fit is achieved in the power values.

For the power curves, it should be noted that the value of the error does not exceed in any case $1 \%$, with the value in almost all the simulation below $0.5 \%$. The largest discrepancies occur for low power values, because in that area, it is more difficult to achieve a good fit of the model.

The model can be simulated with any simulator that enables you to enter the corresponding equations. In our case, we have chosen the PSPICE simulator to perform the application examples. The dissipative parameters can be easily simulated by resistors, the ideal inverter is made with dependent sources and disconnection in abnormal utility conditions is done with controlled switches. The simulation of the reconnection is performed by a transmission line with a delay equal to $T_{R}$. In this way the inverter does not energize the utility line in the time required after the utility service voltage and frequency have recovered to within the specified ranges.

Fig. 18 presents a simulation of the implemented model made in PSPICE. This is a study of the energy generated in a day. The top graph represents the incident radiation, and the bottom one is the energy produced throughout the day by the photovoltaic system. It is noted that its total production is approximately $3.7 \mathrm{~kW} \mathrm{~h}$.

This model has been integrated into a simulation package, which is currently used for educational purposes in the courses offered by our working group [42]. The package allows you to simulate both, stand alone and grid connected PV systems.

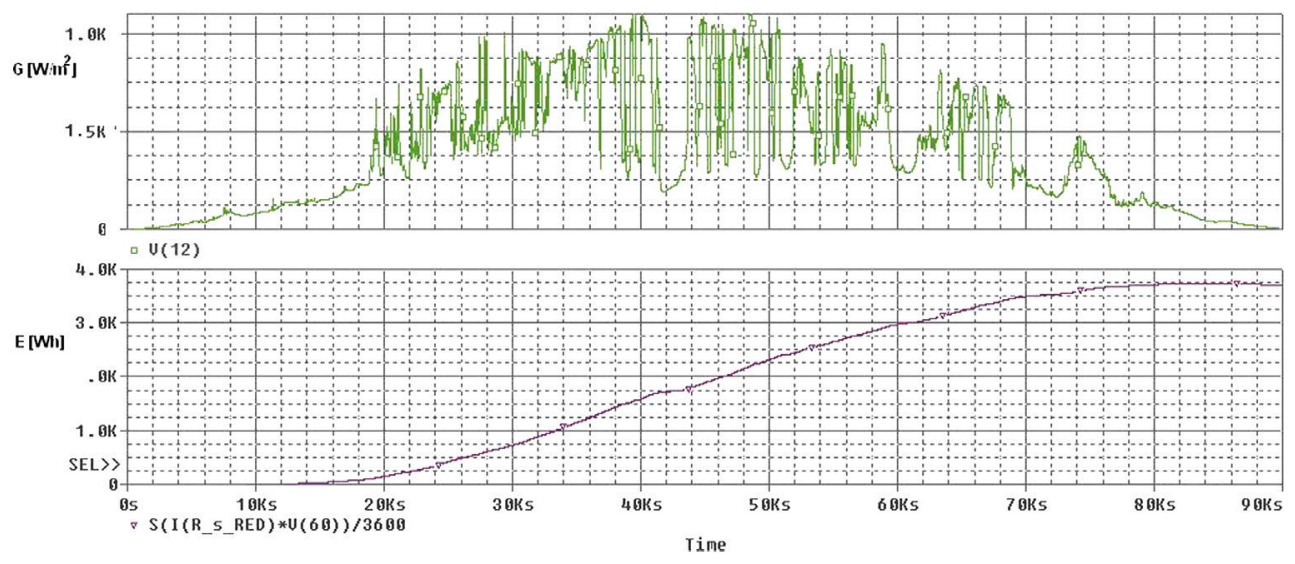

Fig. 18. Energy produced (in Wh) in the course of a day.

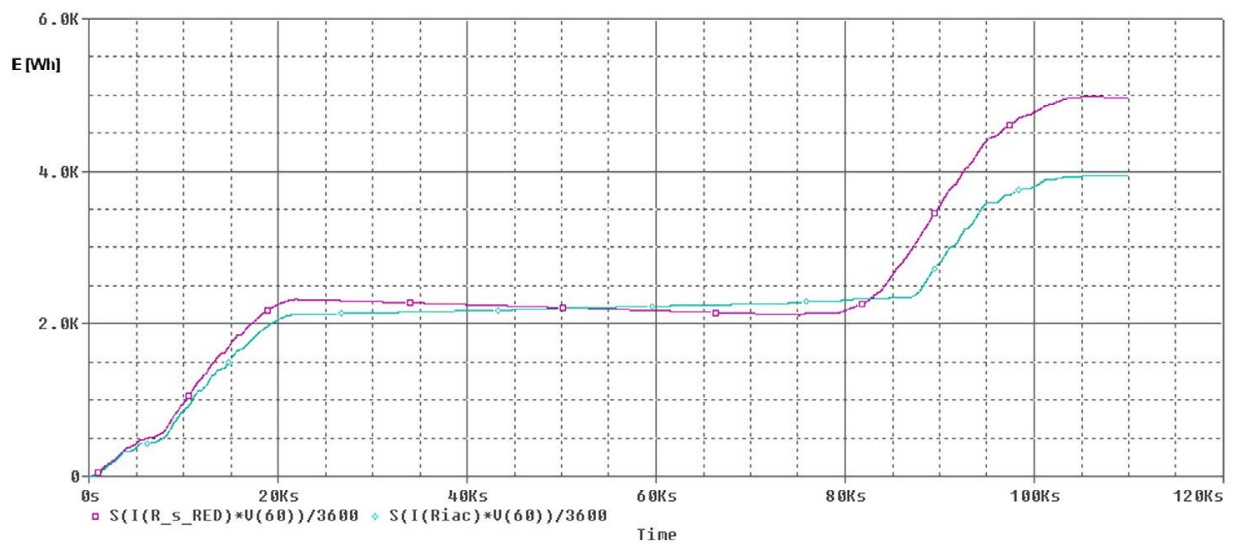

Fig. 19. Energy produced (in Wh) over two days. 


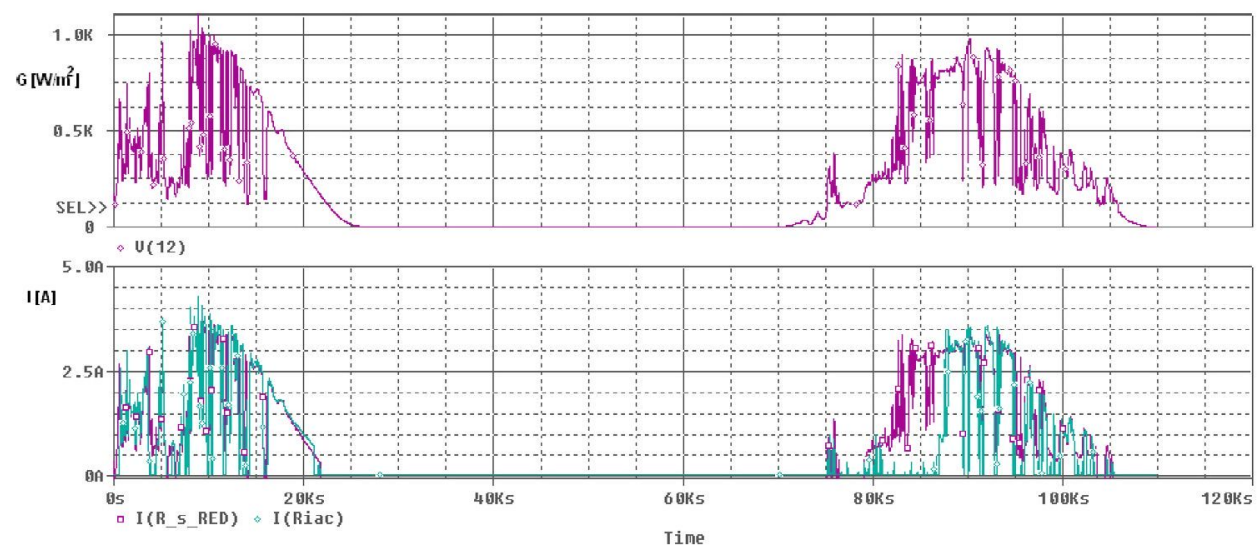

Fig. 20. Radiation (top graph) and output current (lower graph) for the inverter under study.

Another possible use of the model is the diagnosis of PV systems and fault detection by simulation and comparison with real data. In the process of fault diagnosis in a photovoltaic system, it is common that the true cause of the problem is masked by the symptoms of the fault. Using the simulation as a diagnosis tool, you can minimize the number of parameters to measure and use it as a resource to calculate, statistically, the most probable cause of fault.

Fig. 19 represents the energy generated in two days using a photovoltaic system. It can be seen that the energy generated on the first day is virtually identical to the real data curve (in green) and the simulated one (in purple), while the second day both of these graphs differ in approximately $1 \mathrm{~kW} \mathrm{~h}$, being the real the smaller. This suggests that the system has had a problem and has remained disconnected from the utility.

Fig. 20 shows the current output of the inverter. As seen, there has been a disconnection for approximately an hour and a half after that the radiation exceeds the start threshold (see real data curve in green). Subsequent studies attribute the shutdown to a cooling failure, which was revised in this inverter.

\section{Conclusions}

A model that allows simulation of grid-connected PV inverters has been presented. The model is composed of electrical items that simulate the behavior of the electronic system. The model proposes that efficiency, MPPT, and standby consumption are simulated using resistive elements, as shown by Eqs. (10), (13) and (21). The other characteristics are simulated by simple circuits.

The proposed model can be used to characterize any inverter, regardless of their topology and the MPPT control method, against the other models presented in this paper. In addition, it has less parameters than other models. Compared with a similar model, if a correct adjustment of parameter is made, the proposed one presents half the value of error in the efficiency.

A systematic methodology that can be applied to grid-connected PV inverters is proposed. This methodology is useful for the extraction of the parameters included in the model. The proposed method enables the characterization and simulation of any grid-connected inverter. Using this methodology, several inverters have been characterized and it has been proved that the model reflects with great fidelity the actual behavior, with errors less than $3.5 \%$.

The model can be implemented in most of the software packages used in computer simulation, such as SIMULINK, TRNSYS, and SPICE. We present some examples of simulations with PSPICE, which check the accuracy of the model when compared with actual data.

The paper also suggests applications of this model in education and maintenance of PV plants. It can also be a useful tool in the design and implementation of photovoltaic projects. 